\title{
STEPS TOWARDS AN EVOLUTIONARY MEDICINE
}

\author{
M. RIGATO ${ }^{1} \&$ E. TIEZZI ${ }^{2}$ \\ ${ }^{1}$ Department of Physics, Section of Medical Physics, University of Siena, Italy. \\ ${ }^{2}$ Department of Chemical and Biosystems Sciences, University of Siena, Italy.
}

\begin{abstract}
The effects of extremely low frequency electromagnetic fields on living systems are reported and discussed in the framework of evolutionary physics. The discussion is focused on: (a) new epistemological points of view related to the relationship between Prigogine's thermodynamics and evolutionary biology; (b) the relevance of Gödel's theorem on scientific modelling; (c) an attempt to take steps towards an evolutionary medicine. Particular emphasis has been dedicated to the role of sounds, water and clinical response and to the self-organisation action, which can be related to the capacity of human beings to use their own defences to reactivate lost mechanisms.

Keywords: electromagnetic field, irreversibility, uncertainty, water.
\end{abstract}

\section{INTRODUCTION}

As pointed out by Sven Jørgensen [1]: 'if we in few words should give information about the new world picture that has been presented by modern physics during the 20th century, we could apply the following three statements:

1. Everything is relative (Einstein)

2. Everything is uncertain (Heisenberg)

3. Everything is irreversible (Prigogine).'

These three statements have contributed to a completely new perception of the universe, its development and the underlying processes.

The third statement has received less attention in society than the other two, but it has actually changed our view of the world maybe even more than the first two statements. The third statement has indeed introduced the concept of evolution in chemistry and physics.

It was science, in particular Prigogine's thermodynamics, that discovered the narrative beauty of biological evolution, accentuating the evolutionary character of nature. Evolutionary physics, concerned with the study of nature, uses qualities (not only quantities) and time (as did Darwin). In line with Gödel's theorem, evolutionary physics cannot be constrained within a single mathematical structure. In this direction Mae Wan Ho [2] gave an important contribution: " "science is a quest for the most intimate understanding of nature. It is not industry set up for the purpose of validating existing theories and indoctrinating students in the correct ideologies. It is an adventure of the free, enquiring spirit which thrives not so much on answers as unanswered questions. It is the enigmas, the mysteries and paradoxes that take hold of the imagination, leading it on the most exquisite dance", if science is this marvellous dance, the challenge is, today, to aim to a "thermodynamics of organised complexity", having in mind that "there is as yet no science of organised heterogeneity or complexity such as would apply to living systems".'

This is particularly valid for biology and medicine, where feedback mechanisms are also fundamental.

Prigogine suggests the concept of events for the evolutionary behaviour of living systems, ecosystems and human beings.

(C) 2007 WIT Press, www.witpress.com

ISSN: 1743-5242 (paper format), ISSN: 1743-5250 (online), http://journals.witpress.com

DOI: $10.2495 / \mathrm{ECO}-\mathrm{V} 2-\mathrm{N} 4-217-221$ 
In this paper, we discuss the effects of extremely low frequency (ELF) electromagnetic fields in the light of evolutionary physics.

\section{RESULTS AND DISCUSSION}

When a biological system is exposed to an electromagnetic field, the forces induced act on the charges present (ions, free radicals, dipoles) which respond according to their constraints, producing functional modifications in the cell microenvironment.

In clinical experiments conducted by us, we used an ELF (alternating) electromagnetic field at $100 \mathrm{~Hz}$ with sinusoidal waveform. The effective induction was 2-5 gauss at the interpolar midpoint when the latter was at about $30 \mathrm{~cm}$.

We made many observations in cases with different pathologies. We found trophic effects on damaged nerves, modification of pain transduction and transmission, effects on post-herpetic pain with changes in blood levels of beta endorphin and prostaglandins, effects on artery diseases, back pain and sequelae of varicella zoster virus infections, effects on blood concentrations of cAMP, menopausal osteoporosis and primitive carpal tunnel syndrome. The clinical results were similar to those for experimental animals and cell cultures. The benefits obtained were found to be satisfactory but not resolutive. There was no reason to believe that the field code (frequency, intensity, waveform) was optimised.

This finding can also be related to Barbieri's [3] statement that a physical system can increase in complexity only if it has memory and a translation code. Like the genetic codes present in all organisms, the linguistic codes exclusive to our species, and many other codes yet to be deciphered, memories and translation codes must therefore be the fundamental components of all organisms.

This conclusion radically changes our way of looking at the living world, making it impossible to interpret the world as a mere assemblage of objects dominated by the rigid deterministic dialectic of chance and necessity; it confers the world with properties hitherto considered exclusive to the mind or psyche. It is therefore impossible to explain the origin of cell systems without simultaneously explaining the origin of the memories and translation codes they contain [4].

We are left with the problem of determining whether other codes could produce the same or superior benefits. In theory, the next step would be to systematically explore low frequency fields with all possible codes, but this would require an astronomical number of comparisons between homogeneous groups of patients - an impossible task.

Since any comparison would call for a variable field generator, we built an apparatus consisting of a reader of low frequency recorded signals, an amplifier that provided an output, current-modulated according to the pilot signal, and a pair of electromagnets driven by the amplified current. The versatility of the apparatus makes it possible to obtain fields piloted by a wide range of recorded signals. If the reader is fed with the recording of a pure sound at $100 \mathrm{~Hz}$, the field code generated (for a given mean induction) is identical to that of the previous ELF field.

Once the generator had been developed, we had the idea of comparing the effects of the latter ELF field with those of an all-inclusive reference field, e.g. using the pilot signal of white noise. Alternatively, the pilot signal could be a recording of an appropriate piece of music, since a vast variety of codes are generated by different assortments of frequencies, intensities and waveforms during reproduction. This led to a new system which we called the therapeutic application of musically modulated electromagnetic fields (TAMMEF), because the generator enabled the adoption of the operating criterion [5].

It then seemed logical to allow patients to hear the music during treatment, since the effects of the two coherent synchronous stimuli could perhaps reinforce each other, which was done by means of a second amplifier inserted parallel to the first and connected to a speaker or headphones. A further 
step was to inform patients with sufficient intellectual capability about the correlation between the music they were hearing and the electromagnetic field modulated by the musical signal, thus aiming to trigger a further cooperative component, this time psychosomatic, hopefully related to the placebo effect.

We then conducted comparative experiments according to the above criterion. The data obtained to date indicates that the clinical effects obtained by any form of TAMMEF (silent or with listening and in the latter case with or without explanation) cover those previously obtained with the ELF field. Although TAMMEF with listening and explanation seems to give better results (faster response time and/or more positive subjective reports), not enough data has been gathered to draw conclusions.

In another direction, animals' sounds have been investigated using recurrence plots of Prigogine's strange attractors, relating biodiversity to vocal fingerprints [6, 7].

While experimentation continues as described above, a few reflections on epistemological aspects are appropriate.

Benefits that patients experience clinically may be due to two simultaneous, concomitant mechanisms. The first is the local biophysical effect of the applied field, due at least to the evident effects observed in hamsters and cell cultures. The supposed second mechanism has to do with psychophysical complexity. The treatment (ELF or TAMMEF in any form) is presumably not the only cause of the positive clinical response, but since it causes the treated region to emit signals, it stimulates at least partial central reactivation of mechanisms of self-defence and self-repair that act automatically and continuously in healthy subjects. In view of current insights into interactions between the nervous, endocrine and immune systems, it seems reasonable to regard the body's psychophysical unity as a complex homeostatic system.

It follows that the ideal state of health involves efficient self-regulation of all reactive functions which keep that state stable, antagonising all attempts to alter it. A state of illness occurs when the central control breaks down and defensive measures are no longer promoted and coordinated appropriately.

According to this view, the output of the cell microenvironment concomitant with local modifications induced could stimulate reactivation of central control if the system is favourably disposed. In such a model, the system would selectively recognise corrective stimuli among the signals reaching it and would respond to them through functional restoration; it would react to other signals through mechanisms of self-adaptation which maintain homeostasis. If so, the effects observed do not demonstrate a revolutionary therapy of great efficacy but simply suggest that such a system is possible and should be investigated.

A working hypothesis in this direction is that when the body is ill it has the capacity to heal without external help by reactivating the mechanisms that failed and that reduced the efficacy of self-defence. Reactivation can, however, be stimulated psychosomatically by external signals. This view is sustained by well-known effects induced by emotions that can translate into violent physiological reactions, such as vomiting, diarrhoea, convulsions, fainting and even heart attack, as well as subtle psychosomatic effects such as those presumably induced by TAMMEF treatment.

\section{CONCLUSION}

As St Augustine observed: 'What happens never breaks the laws of nature, but can be contrary to what we believe about those laws.' This is the reason why we do new research.

On one hand, we are prisoners because we are made of matter and cannot escape the laws of matter by changing or eluding them. On the other hand, we are operators capable of improving our state because we can investigate and discover the laws and exploit them to our advantage. Use of this 
faculty is always, however, subordinate to the condition of considering all observational data to be natural.

In addition, unconditional prior acceptance of the naturalness of any event ought not to offend scientists who are believers, because the image arising does not detract from the grandiosity of a metaphysical design that may have been made once, at the beginning, when universal evolution was established. Nature is not inscrutable. The inscrutability is the reason why nature has been designed this way.

Niels Bohr said: 'I do not believe in superstition, but they say that a horse-shoe bat is good also for people who do not believe in superstition.'

Drawing conclusions in order of logical consequentiality, let us begin by recognising that if certain phenomena seem inexplicable, this only means that the world is not as we see it or believe it to be.

Prigogine emphasises that 'we now know that far from equilibrium (living systems), new types of structures may originate spontaneously. In far-from-equilibrium conditions we may have transformation from disorder, from thermal chaos, into order. New dynamic states of matter may originate, states that reflect the interaction of a given system with its surroundings. We have called these new structures dissipative structures to emphasise the constructive role of dissipative processes in their formation.' It is the emergence of novelties.

For example, as pointed out by Prigogine, low-entropy dissipative structures have coherent supramolecular character, which leads to spectacular new manifestations, such as biochemical cycles involving oscillatory enzymes. These spatiotemporal structures arise from the non-linear dynamics of such phenomena. This is particularly related to the supramolecular structure of water. The evolution of aqueous systems vastly exceeds typical 'molecular' time-scales. This, together with very-lowconcentration and electromagnetic-field effects, suggests that water and dilute aqueous solutions can be regarded as self-organising systems.

At the macrolevel, the behaviour of water is related to biodiversity, the core of biological evolution.

We assert here that the action of drugs and medicines is mediated by the supramolecular structure of water and that the supramolecular structure of water is related to the formation of dissipative structures and to their self-organisation.

Going back to the effect of ELF in the psychophysical domain, at the very least we know that low frequency electromagnetic fields have antalgic and therapeutic effects which are satisfactory but not resolutive. On one hand, the part played by psychosomatic components can be presumed but is not ascertained, and may be reinforced to relatively optimal levels by the integrated TAMMEF system. At the very most, immediate violent effects, mostly harmful, are known to be produced by emotions through undoubtedly psychosomatic mechanisms; on the other hand, beneficial, resolutive, equally immediate and violent effects, like miraculous healing due to unknown mechanisms, may be linked to individual psychosomatic processes or the result of multiple interpersonal interactions. On this subject, we can recognise that when we open our minds to embrace views that qualify as suggestion - placebo effect, psychosomatic effects, polypsychism, interpersonal interactions and so forth - rigorously, we are simply assigning names to unknowns.

In conclusion, at the direct observational level we find that the laws of nature enable healing processes that can by triggered by induction, whatever the urges to induce may be. Thus, if integrated TAMMEF treatment (with listening and explanation) is unequivocally more efficacious than silent TAMMEF treatment (field modulated according to the same pilot signal but without listening or explanation), this observation can only be a starting point for research into sufficient conditions under which bodies that have fallen ill are induced to tune their homeostatic reactivity and recover the previous state of health by their own means. 
Until only a few centuries ago, it could not be imagined that nature hid properties that could be used to build the powerful instruments we have today, such as radio in real time, noninvasive exploration of the human body and laser microsurgery. Electromagnetic oscillations existed and could have been used even before their existence was suspected. Thus, we may well ask if we have good reason to be reticent about tackling with cold scientific rigour the possibility that other natural properties, unbeknown to us, open the way to self-therapeutic stimulation, with effects just as fast and resolutive as those we observe as spectators in cases of spontaneous miraculous healing. The answer is evident. Is it thus possible to conceive evolutionary medicine?

\section{REFERENCES}

[1] Jørgensen, S., Foreword in Tiezzi, E., Steps Towards an Evolutionary Physics, WIT Press: Southampton, 2006.

[2] Ho, M.W., The Rainbow and the Worm, World Scientific Publ. Co.: Singapore, 1998.

[3] Barbieri, M., The Organic Codes. The Birth of Semantic Biology, PeQuod: Ancona, 2001.

[4] Fondi, R., Evolutionism and holism: two different paradigms for the phenomenon of biological evolution. International Journal of Ecodynamics, 1(3), pp. 284-297, 2006.

[5] Rigato, M., Battisti, E., Fortunato, M. \& Giordano, N., Comparison between the analgesic and therapeutic effects of a musically modulated electromagnetic field (TAMMEF) and those of a $100 \mathrm{~Hz}$ electromagnetic field: blind experiment on patients suffering from cervical spondylosis or shoulder periarthritis. Journal of Medical Engineering \& Technology, 26(6), pp. 253-258, 2002.

[6] Tiezzi, E., Steps Towards an Evolutionary Physics, WIT Press: Southampton, 2006.

[7] Facchini, A., Kantz, H. \& Tiezzi, E., Recurrence plot analysis of nonstationary data: the understanding of curved patterns. Phys. Rev. E, 72, 21915, 2005. 